See discussions, stats, and author profiles for this publication at: https://www.researchgate.net/publication/264862078

\title{
A Comparison of Methodologies for Intelligent Computing Used to Integrity Analysis of a Structure Aeronautic
}

Article in Applied Mechanics and Materials · August 2014

Dol: 10.4028/www.scientific.net/AMM.610.253

\section{CITATIONS}

5 authors, including:

Fernando Parra dos Anjos Lima

Instituto Federal de Educação, Ciência e Tecnologia de Mato Grosso (IFMT) 43 PUBLICATIONS 151 CITATIONS

SEE PROFILE

A. Simone S. F. Souza

Universidade do Estado do Mato Grosso (UNEMAT)

30 PUBLICATIONS 103 CITATIONS

SEE PROFILE
READS

28

- Fábio Roberto Chavarette

São Paulo State University

89 PUBLICATIONS 237 CITATIONS

SEE PROFILE

Mara Lúcia M. Lopes

São Paulo State University

34 PUBLICATIONS 203 CITATIONS

SEE PROFILE

Some of the authors of this publication are also working on these related projects:

Graduation course completion work View project 


\title{
A Comparison of Methodologies for Intelligent Computing Used to Integrity Analysis of a Structure Aeronautic
}

\author{
Fernando Parra dos Anjos Lima ${ }^{1, a}$, Fábio Roberto Chavarette ${ }^{3, b}$, \\ Simone Silva Frutuoso de Souza, c, Adriano dos Santos e Souza ${ }^{1, e}$, \\ Mara Lúcia Martins Lopes ${ }^{3, \mathrm{e}}$, \\ ${ }^{1}$ Mechanical Engineering Department, Faculty of Engineering of Ilha Solteira (FEIS) \\ ${ }^{2}$ Electrical Engineering Department, Faculty of Engineering of Ilha Solteira (FEIS) \\ ${ }^{3}$ Mathematics Department, Faculty of Engineering of Ilha Solteira (FEIS) \\ UNESP - Univ.Estadual Paulista, Av. Brasil, 56, 15385-000, Ilha Solteira, SP, Brazil \\ aengfernandoparra@gmail.com, ${ }^{\text {b}}$ fabioch@mat.feis.unesp.br, 'simonefrutuoso.mat@gmail.com, \\ deng.adrianosouza@gmail.com, ${ }^{\mathrm{e}}$ mara@mat.feis.unesp.br
}

Keywords: Analysis of Structural Integrity, Aeronautical Structures, Artificial Immune Systems, Artificial Neural Networks.

\begin{abstract}
This article presents the application and comparison of two techniques for intelligent computing to perform the analysis of the structural integrity of an aircraft structure. In this context, a ARTMAP-Fuzzy neural network and immunological negative selection algorithm are used in the identification and characterization of structural failure. The main application of these methodologies is to assist in the inspection of aircraft structures aiming at detecting and characterize flaws and decision making. To evaluate the methodology was performed modeling and simulation of signals from a numerical model using an aluminum beam. We performed a comparative analysis of methodologies, proving the efficiency of intelligent methods in the analysis of structural integrity. The results obtained by the method show efficiency, robustness and accuracy. To Evaluate the methodology was Performed modeling and simulation of signals from the numerical model using an aluminum beam. We performed a comparative analysis of methodologies, proving the efficiency of intelligent methods in the analysis of structural integrity. The results Obtained by the methods show efficiency, robustness and accuracy.
\end{abstract}

\section{Introduction}

Despite the technological advancement and the development of new materials and aircraft structures, many investments are still employed in structural analysis, with the aim of ensure economic, security and verify the integrity of the structure.

In aircraft structural failure can alter the dynamic behavior of the structure, causing a significant variation in the spatial parameters of the structure, reducing the stiffness and structural mass, and also an increased damping in most cases. Failures can occur as a consequence of various factors, such as component wear, cracks, loosening of bolted connections, or simply the combination of these elements. With the variation of the spatial parameters, the structure can present variations dynamic parameters, such as response functions, resonance frequencies, damping ratio and eigen modes of vibration of the structure [10].

In the current literature we found some studies that use methodologies to conduct analysis of structural integrity in this way, stand out the following work: in the article [6] used the method of the electromechanical impedance for monitoring aerospace structures with active piezoelectric sensors. A system for identifying and locating damage in an airplane wing was proposed in [9]. In [11] was proposing a multinodal genetic algorithm to diagnose damage in a steel truss bridge.

In this paper, we present a comparison between two methodologies for monitoring and analysis of aeronautical structures. With the acquisition and processing of signals, applies a ARTMAP-Fuzzy neural network [1] negative selection immune algorithm (NSA) [5] to identify and characterize flaws. To evaluate the methodologies used a data base of simulated signals from a 
numerical model of a beam of aluminum, which emulates a wing structure of an aircraft. The beam was modeled using the finite element method and simulated in Matlab® [8].

\section{Negative Selection Algorithm (NSA)}

The negative selection algorithm (NSA) has been proposed by [5] to detect changes in system state, and is based on negative selection of $\mathrm{T}$ cells within the thymus. This algorithm is the discrimination proper and non-proper cells. The algorithm can be described in the following steps [4]:

1) Censor Phase

a. Define the proper set of chains $(\mathrm{S})$ to be protected;

b. Generate random chains and evaluate the affinity (Match) between each of them and the proper chains. If the affinity is higher than a stipulated threshold, reject the chain. Otherwise, store it in a set of detectors (R);

2) Monitoring

a. Be the set of chains to be protected (protected chains), evaluate the affinity between each of them and the set of detectors. If the affinity is higher than a predetermined threshold, then a non-proper element is identified;

The censor phase of NSA basically consists in generating a set of detectors. The detectors are analogous to $\mathrm{T}$ type cells matured able to recognize pathogenic agents. The monitoring phase consists in monitoring a system to identify a change in the behavior of the same, and thus classify this change using the set of detectors created in censor phase [7]. In this study we used the partial matching criterion. The rate of affinity represents the degree of similarity needed for the marriage occurs between the two standards and is defined by the following relationship [3]:

$$
T A f=\left(\frac{A n}{A t}\right) * 100
$$

being: TAf the afinity rate, $A n$ the number of chains in normal problem and $A t$ the total number of chains in problem. The equation (1) proposes a statistical relationship between all samples of the problem to the calculation of the affinity rate. In (2) represents a way of quantifying the overall affinity between patterns [7]:

$$
A f_{T}=\frac{\sum_{i=1}^{L} V c}{L} * 100
$$

Where in $A f_{T}$ the $\%$ affinity between patterns analyzed, $L$ the total number of variables and $V c$ the variables matching. If $A f_{T}>=T A f$ matching occurs between the two patterns (are considered similar).

\section{ARTMAP-Fuzzy Artificial Neural Network}

The ARTMAP-Fuzzy artificial neural network (ANN) has an architecture based on adaptive resonance theory, ie, belongs to the ART family (Adaptive Resonance Theory) [2]. This network corresponds to a supervised learning system consists of a pair of modules of adaptive resonance theory, $A R T_{a}-F u z z y$ and $A R T_{b}-F u z z y$, which receive and classify the inputs $\left(I_{a}\right)$ and desired outputs $\left(I_{b}\right)$ respectively, and the inter- $A R T$ associative memory module checks the matching of inputs and outputs with the existing categories [1].

The ARTMAP-Fuzzy architecture has three key parameters for its development, being [1]: Parameter choice $\alpha(\alpha>0)$ : Operates in the category selection, Rate Training $\beta\left(\beta \in\left[\begin{array}{ll}0 & 1]\end{array}\right)\right.$ : Controls 
the speed of adaptation of the network and Parameter monitoring $\left(\rho_{a}, \rho_{b}\right.$ e $\left.\rho_{a b} \in\left[\begin{array}{ll}0 & 1\end{array}\right]\right)$ : Controls the resonant network, namely the parameter responsible for the number of categories created.

If $\rho$ has a large value, the neural network becomes more selective reducing its generalization capability. If $\rho$ has a small value, it reduces the number of categories created, increasing the generalization capability of the ARTMAP-Fuzzy network.

\section{Modeling and Simulations}

To evaluate the methods proposed in this work, we used the finite element method to model an aluminum beam clamped-free condition in discretized with 10 finite elements with two degrees of freedom each. The material properties used are: modulus of elasticity, $\mathrm{E}=700 \mathrm{GPa}$, density, $\gamma=$ $2710 \mathrm{~kg} / \mathrm{m}^{3}$. The dimensions are $500 \mathrm{~mm}$ long, $25 \mathrm{~mm}$ wide and $5 \mathrm{~mm}$ thick. Fig. 1 (a) illustrates the patterned beam.

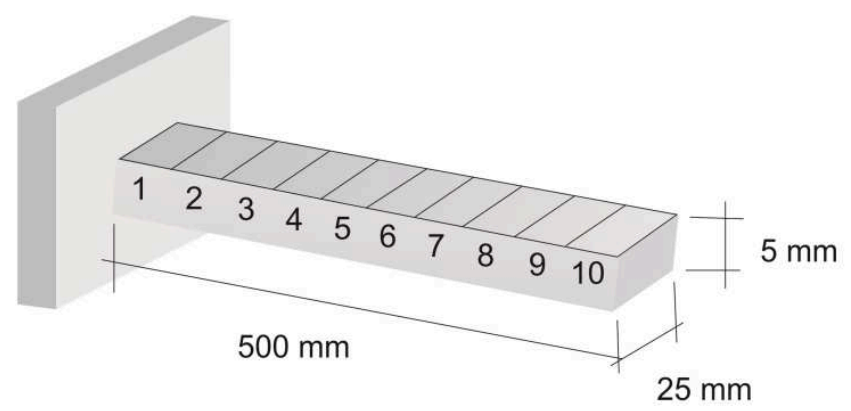

(a)

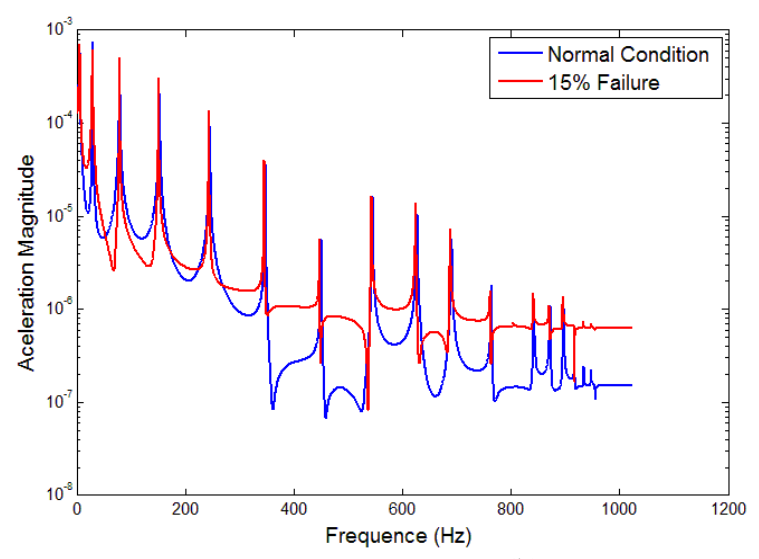

(b)

Fig. 1: (a) Beam modeled, (b) Simulated signal.

From the beam model were performed several simulations with different percentages of wear and locations of faults. The generated database consists signal captured by an accelerometer fixed to the beam. In all simulations the beam was excited in the 3rd degree of freedom (finite element two) and the signal was captured on the 19th degree of freedom (finite element ten). Thus, were simulated 540 signals in the structure, 400 without wear (base-line condition) and 140 signs with wear (structural failure). The signals at failure were simulated in wear levels 1, 5, 10, 15, 20, 25 and $30 \%$, begging each failure has been positioned at two different locations in finite element 3 and 5 with 10 simulated signals at each location. Fig. 1 (b) presents two signals that had been captured in the simulations, a $15 \%$ failure and another under normal conditions.

\section{Proposed Methodology}

Systems integrity analysis of aeronautical structures proposed in this work are composed of three main modules: data acquisition, obtaining knowledge (training or censor) and the diagnosis, as shown in Figure 2:

ARTMAP-Fuzzy Artificial Neural Network. The data acquisition module comprises the experimental part for capturing the signals on the mechanical structure, such as sensors, actuators, accelerometers, etc. In the training module and the weights are adjusted created the categories (knowledge) that will be used in the diagnosis of the data by ARTMAP-Fuzzy neural network. In this phase the network is trained using a data set is generated and a number of categories equals the number of input signals. The diagnostic module signals the test set are presented to the network, and each signal is compared with the knowledge (categories created in the training phase) obtained by ARTMAP-Fuzzy neural network and so when there is a marriage between the patterns classified category in the displayed signal the ARTMAP-Fuzzy network. Obtained category corresponding to a given pattern is thus identified and classified failure in the structure. 
Negative Selection Algorithm (NSA). The data acquisition module is the same ARTMAP Fuzzy neural network. The censor module the NSA is used to generate a set of fault detectors to be used in the diagnosis data. In the case of NSA the signals at faults are detected through evaluation and verifying marriage to the set of proper detectors (base-line signals) thus carries out the detection of signals in fail discrimination on the basis proper and non-proper. If the signal identified is proper the same is instantly classified as the normal condition of the structure. Otherwise, the signal identified for a non-proper (signal failure) carried out an evaluation of this signal, comparing it with the set of failure detectors, thus to occur wedding sign with a class of detectors, the signal is classified as a class of detector activated.

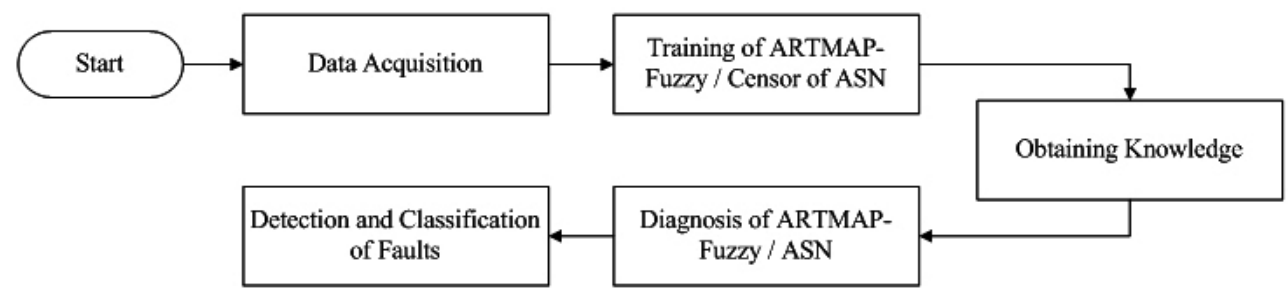

Fig 2: Functioning of the methodology.

\section{Applications and Results}

In this section we present the results obtained with the application the methods in the database simulated. All tests were performed using a PC Intel Core 2 Duo $1.9 \mathrm{GHz}, 2 \mathrm{~GB}$ of RAM, operating system Windows 7 Ultimate 32 bits. The algorithm was developed in Matlab® [8].

For the ARTMAP-Fuzzy neural network the parameters used in the training process and diagnosis were: $\alpha=0,1, \beta=1, \rho_{a}=0,99, \rho_{b}=0,99$ e $\rho_{a b}=1$. For the NSA was used as a parameter an affinity rate with a fixed value of $74 \%$ (calculated with equation (1)). To evaluate the ARTMAP-Fuzzy neural network the database wass divided in two parts (70\% training and $30 \%$ test). To evaluate the NSA, were generated three sets of detectors (I, II and III) using 10\%, 20\% and $30 \%$ of available signals, totaling 54, 108 and 162 detectors, respectively. The database has seven different patterns of faults. The signals obtained by the frequency acquisition module representing the input signals of both methods. Table 1 shows the results obtained with the two systems when applied to the data set .

Table 1 - Results obtained by the method.

\begin{tabular}{cccccc}
\hline \multirow{2}{*}{ Diagnosis } & \multicolumn{3}{c}{ NSA } & \multicolumn{3}{c}{ ARTMAP-Fuzzy } \\
\cline { 2 - 6 } & $I$ & $I$ & $I I$ & Training & Test \\
\hline Samples tested & 540 & 540 & 540 & 398 & 142 \\
\hline Ratings correct & 533 & 538 & 540 & 398 & 142 \\
\hline Ratings wrongs & 7 & 2 & 0 & 0 & 0 \\
\hline Accuracy $(\%)$ & 98.70 & 98,62 & 100.00 & 100.00 & 100.00 \\
\hline Time (ms) & 163,5 & 162,10 & 158,00 & 735,23 & 146,25 \\
\hline
\end{tabular}

The parameters used in the methods were obtained empirically by testing. The result was obtained by cross-reference test, performed twenty times to ensure accuracy of results. Both systems identified and classified $100 \%$ of the signals failed in its best configuration. We note that for the ARTMAP-Fuzzy neural network the more knowledge obtained in training, the more efficient the process of diagnosis. As fo the NSA, the greater the number of detectors, better diagnosis phase tracking. By comparing the proposed methods we obtain that both feature precision, robustness and efficiency, therefore, have identified and classified all faults. In relation to the processing time is greater than ARTMAP-Fuzzy network, however, both can be applied in real time, facilitating decision-making.

It is noteworthy that the two methods discussed are inspired by completely different theories, however, have accuracy, efficiency and good performance. Thus, either of the two methods can be applied to the analysis of aeronautical structures, which present good results. 


\section{Conclusion}

In this paper, we propose two new approaches based on intelligent computing for analysis of aircraft structures, in this context we used an ARTMAP-Fuzzy artificial neural network and NSA, that showed good results, achieving $100 \%$ accuracy for the best configuration of both methods. Both the training and the censor phase, require more computational time, however are executed off-line, not causing harm to the system. The diagnostic data is performed with time less than 160 milliseconds, what allows methods to be used in real time. Thus, we conclude that the intelligent computing provides efficiency, reliability, robustness and accuracy in the analysis of aircraft structural integrity. This work contributes to the research line, presenting a comparison of two efficient methods employing intelligent techniques.

\section{Acknowledgments}

The authors thanks the CAPES and CNPQ (proc. $n^{\circ} 301769 / 2012-5$ ) for a financial supports.

\section{References}

[1] G. A. Carpenter; S. Grossberg, N. Markuzon; J. H. Reynold and D. B. Rosen. Fuzzy ARTMAP: A neural network for incremental supervised learning of analog multidimensional maps. IEEE Transactions on Neural Network, vol. 3, n. 5, p. 689-713, (1992).

[2] G. A. Carpenter and S. Grossberg. A massively parallel architecture for a self-organizing neural pattern recognition machine. Computer Vision, Graphics, and Image Processing, p. 54-115, (1987).

[3] D. W. Bradley and A. M. Tyrrell. Immunotronics - Novel Finite-State-Machine Architectures with Built-In Self-Test Using Self-Nonself Differentiation. IEEE Transactions on Evolutionary Computation.Vol. 6, pp. 227-238 (2002).

[4] L. N. de Castro and J. TIMMIS. "Artificial Immune Systems: A New Computational Intelligence Approach”, Springer. 1st edition, 2002.

[5] S. Forrest; A. Perelson; L. Allen and R. Cherukuri. Self-Nonself Discrimination in a computer, Proc. do IEEE Symposium on Research in Security and Privacy, pp. 202-212 (1994).

[6] V. Giurgiutiu. Tuned lamb wave excitation and detection with piezoelectric wafer active sensors for structural health monitoring. Journal of Intelligent Material Systems and Structures, USA, v. 16, n. 4, p. 291-305, (2005).

[7] F. P. A. Lima; A. D. P. Lotufo and C. R. Minussi. Artificial Immune Systems Applied to Voltage Disturbance Diagnosis in Distribution Electrical Systems, PowerTech-2013, Grenoble, France, Junhe-2013, 6 p, (2013).

[8] Matlab (2011). 7.8 Version, Mathworks Company.

[9] T. Shen; F. Wan; B. Song; Y. Wu. Damage Location and Identification of the Wing Structure With probabilistic neural Networks. Prognostics \& System Health Management Conference (PHM 2011). p. 1-6, (2011).

[10] S. Zheng; X. Wang; L. Liu. Damage detection in composite materials based upon the computational mechanics and neural networks. In: European Workshop on Structural Health Monitoring, Munich, p. 609-615 (2004).

[11]F. L. Wang; T. H. T. Chan; D. P. Thambiratnam and A. C. C. Tan. Damage Diagnosis for Complex Steel Truss Bridges Using Multi-Layer Genetic Algorithm. Journal of Civil structural Health Monitoring, Springer-Verlag, p. 117-217, (2013). 
Mechanics, Mechatronics, Intelligent System and Information Technology

10.4028/www.scientific.net/AMM.610

A Comparison of Methodologies for Intelligent Computing Used to Integrity Analysis of a Structure Aeronautic

10.4028/www.scientific.net/AMM.610.253 